\title{
Protein $O$-fucosyltransferase 1: A potential diagnostic marker and therapeutic target for human oral cancer
}

\author{
SATOSHI YOKOTA ${ }^{1}$, KATSUNORI OGAWARA ${ }^{3}$, RYOTA KIMURA ${ }^{1}$, FUMIE SHIMIZU ${ }^{1}$, TAKAO BABA ${ }^{1}$, \\ YASUYUKI MINAKAWA ${ }^{1}$, MORIHIRO HIGO ${ }^{3}$, ATSUSHI KASAMATSU ${ }^{3}$, YOSUKE ENDO-SAKAMOTO ${ }^{3}$, \\ MASASHI SHIIBA $^{2}$, HIDEKI TANZAWA ${ }^{1,3}$ and KATSUHIRO UZAWA ${ }^{1,3}$
}

Departments of ${ }^{1}$ Clinical Molecular Biology and ${ }^{2}$ Clinical Oncology, Graduate School of Medicine, Chiba University, Chuo-ku, Chiba 260-8670; ${ }^{3}$ Division of Dentistry and Oral-Maxillofacial Surgery,

Chiba University Hospital, Chuo-ku, Chiba 260-8677, Japan

Received July 19, 2013; Accepted September 2, 2013

DOI: $10.3892 /$ ijo.2013.2110

\begin{abstract}
Protein $O$-fucosyltransferase 1 (POFUT1) is the enzyme that adds $O$-fucose through $O$-glycosidic linkage to conserved serine or threonine residues in the epidermal growth factor-like repeats of a number of cellular surface and secreted proteins. Our previous study using microarray technology showed that significant upregulation of POFUT1 occurs in oral squamous cell carcinoma (OSCC)-derived cell lines compared to human normal oral keratinocytes. The aim of the present study was to examine the status of POFUT1 mRNA and protein expression in OSCC-derived cell lines and human primary OSCCs. POFUT1 mRNA was upregulated significantly $(\mathrm{P}<0.05$ for both comparisons) in five OSCC-derived cell lines and primary OSCCs using quantitative reverse transcriptase-polymerase chain reaction. Immunohistochemistry data indicated that POFUT1 protein expression levels were consistent with mRNA expression status in OSCC-derived cell lines and primary OSCCs. Furthermore, POFUT1 expression status was correlated significantly $(\mathrm{P}=0.048)$ with the primary tumor size. The proliferation of POFUT1 knockdown cells was inhibited significantly compared with that of control cells. These results indicated that POFUT1 expression can contribute to cancer progression and that POFUT1 may serve as a diagnostic marker and a therapeutic target for OSCCs.
\end{abstract}

Correspondence to: Dr Katsuhiro Uzawa, Department of Clinical Molecular Biology, Graduate School of Medicine, Chiba University, 1-8-1 Inohana, Chuo-ku, Chiba 260-8670, Japan

E-mail: uzawak@faculty.chiba-u.jp

Dr Katsunori Ogawara, Division of Dentistry and Oral-Maxillofacial Surgery, Chiba University Hospital, 1-8-1 Inohana, Chuo-ku, Chiba 260-8677, Japan

E-mail: katsunori.ogawara@faculty.chiba-u.jp

Key words: oral squamous cell carcinoma, protein $O$-fucosyltransferase 1, POFUT1, proliferation assay

\section{Introduction}

Oral squamous cell carcinoma (OSCC) is a frequently occurring neoplasm that is usually aggressive and has a poor prognosis. Improvements in specificity and sensitivity of diagnosis and prognosis depend on the elucidation of the biologic and molecular mechanisms underlying carcinogenesis (1). The availability of biomarkers of malignancy would be a key factor for monitoring cancer recurrence and evaluating the efficacy of novel treatments. The accumulation of genetic alterations during carcinogenesis is currently known but still largely unexplored (2-5).

We previously reported gene expression profiling of OSCCs using microarray analysis to identify genes associated with oral carcinogenesis (6). The analysis showed that protein $O$-fucosyltransferase 1 (POFUT1) expression was significantly upregulated.

POFUT1 attaches $O$-fucose through $O$-glycosidic linkage to conserved serine or threonine residues in the epidermal growth factor-like (EGF-like) repeats of a number of cellular surface and secreted proteins (7). In the present study, further analysis of the status of POFUT1 expression showed that POFUT1 expression increased in OSCCs compared with normal oral tissues. Based on these data, we proposed that POFUT1 may be a key regulator of tumor progression in OSCCs.

\section{Materials and methods}

OSCC-derived cell lines and tissue samples. The human OSCC cell lines (Ho-1-u-1, KOSC2, HSC-4, HSC-3 and HSC-2) were purchased from the Human Science Research Resources Bank, Osaka, Japan. Primary cultured human normal oral keratinocytes (HNOKs) served as normal controls (8-17). All cells were maintained in Dulbecco's modified Eagle's medium (DMEM)/F-12 Ham (Sigma, St. Louis, MO, USA) supplemented with $10 \%$ fetal bovine serum (FBS) (Sigma) and $50 \mathrm{U} / \mathrm{ml}$ penicillin and streptomycin (Sigma) in a humidified $5 \% \mathrm{CO}_{2} /$ air atmosphere at $37^{\circ} \mathrm{C}(18-20)$.

One hundred and twenty-eight pairs of primary OSCC samples and corresponding normal oral epithelial tissues were obtained intraoperatively at Chiba University Hospital, 
Chiba, Japan. All patients provided informed consent for use of the protocol, which the institutional review board of Chiba University reviewed and approved. The resected tissues were divided into two parts, one of which was frozen immediately and stored at $-80^{\circ} \mathrm{C}$ until RNA isolation and the second was fixed in $20 \%$ buffered formaldehyde solution for pathologic diagnosis and immunohistochemistry (IHC). Histopathological diagnosis of each tissue was performed according to the World Health Organization criteria by the Department of Pathology, Chiba University Hospital. Clinicopathological staging was determined according to the tumor-node-metastases classification of the International Union against Cancer. All OSCC samples were confirmed histologically and checked to ensure the presence of tumor in $>90 \%$ of specimens.

Preparation of $c D N A$. Total RNA was isolated using TRIzol reagent (Invitrogen, Carlsbad, CA, USA) according to the manufacturer's instructions. cDNA was generated from $5 \mu \mathrm{g}$ of total RNA using Ready-To-Go You-Prime First-Strand Beads (GE Healthcare, Buckinghamshire, UK) and oligo(dT) primer (Hokkaido System Science, Sapporo, Japan) according to the manufacturer's instructions.

mRNA expression analysis. Real-time quantitative reverse transcriptase-polymerase chain reaction (qRT-PCR) was performed to evaluate the expression level of POFUT1 mRNA in the OSCC-derived cell lines and HNOKs. Primary OSCCs and paired specimens of normal oral tissues obtained from 128 patients also were evaluated. The expression levels were determined using primers and probes that were designed using the Universal Probe Library (Roche Diagnostics, Mannheim, Germany) following the manufacturer's recommendations. The primer sequences used for qRT-PCR were: POFUT1, forward 5'-CTGATGACCCGATGGTAAGC-3'; reverse 5'-AAG CCTCCTTTCACCAACCT-3'; and universal probe no. 78. All qRT-PCR analyses were performed using a LightCycler ${ }^{\circledR} 480$ PCR system (Roche Diagnostics). Amplifications were initiated by a 10 -min pre-incubation at $95^{\circ} \mathrm{C}$, followed by 45 cycles of $10 \mathrm{sec}$ at $95^{\circ} \mathrm{C}$ for template denaturation, $30 \mathrm{sec}$ at $55^{\circ} \mathrm{C}$ for primer annealing/extension and cooling for $30 \mathrm{sec}$ at $40^{\circ} \mathrm{C}$. The transcript amount for POFUT1 was estimated from the respective standard curves and normalized to the glyceraldehyde-3-phosphate dehydrogenase (GAPDH) (forward, 5'-CAT CTCTGCCCCCTCTGCTGA-3'; reverse, 5'-GGATGACCTT GCCCACAGCCT-3'; and universal probe no. 60) transcript amount determined in corresponding samples.

Protein extraction. The cells were washed twice with cold phosphate buffered saline (PBS) and centrifuged at $500 \mathrm{x} \mathrm{g}$. The cell pellets were incubated at $4^{\circ} \mathrm{C}$ for $30 \mathrm{~min}$ in a lysis buffer [7 M urea, $2 \mathrm{M}$ thiourea, $4 \% \mathrm{w} / \mathrm{v}$ CHAPS and $10 \mathrm{mM}$ Tris ( $\mathrm{pH}$ 7.4)] with the proteinase inhibitor cocktail (Roche Diagnostics). The protein concentration was measured with a protein assay (Bio-Rad Laboratories, Hercules, CA, USA).

Western blot analysis. Cytoplasmic and nuclear proteins $(50 \mu \mathrm{g})$ were separated by sodium dodecyl sulfate polyacrylamide gel electrophoresis in $4-12 \%$ gel, transferred to nitrocellulose membranes and blocked for $1 \mathrm{~h}$ at room temperature in Blocking One (Nacalai Tesque, Tokyo, Japan).
The membranes were incubated with anti-POFUT1 polyclonal antibody (OriGene Technologies, Rockville, MD, USA) and GAPDH (Santa Cruz Biotechnology, Santa Cruz, CA, USA) antibodies for $4 \mathrm{~h}$ at room temperature. The membranes were washed with $0.1 \%$ Tween-20 in Tris-buffered saline, incubated with secondary antibodies and coupled to horseradish peroxidase-conjugated anti-rabbit IgG (Promega, Madison, WI, USA) in Blocking One for $1 \mathrm{~h}$ at room temperature. The proteins were detected by SuperSignal Chemiluminescent substrate (Thermo, Waltham, MA, USA). Finally, immunoblotting results were visualized by exposing the membrane to a cooled CCD camera system (Light Capture ${ }^{\circledR}$, ATTO, Tokyo, Japan). Signal intensities were quantitated using the CS Analyzer ${ }^{\circledR}$ version 3.0 (ATTO).

$I H C$. IHC was performed on $4-\mu \mathrm{m}$ sections of paraffinembedded specimens using rabbit anti-POFUT1 polyclonal antibody (OriGene Technologies). Briefly, after deparaffinization and hydration, the endogenous peroxidase activity was quenched by 30-min incubation in a mixture of $0.3 \%$ hydrogen peroxide solution in $100 \%$ methanol. The sections were blocked for $2 \mathrm{~h}$ at room temperature with $1.5 \%$ blocking serum (Santa Cruz Biotechnology) in PBS before reacting with anti-POFUT1 antibody (1:1,000 dilution) at $4^{\circ} \mathrm{C}$ in a moist chamber overnight. Upon incubation with the primary antibody, the specimens were washed three times in PBS and treated with Envision reagent (Dako, Carpinteria, CA, USA) followed by color development in 3,3'-diaminobenzidine tetrahydrochloride (Dako). Finally, the slides were counterstained lightly with hematoxylin, dehydrated with ethanol, cleaned with xylene and mounted. Non-specific binding of the antibody to proteins other than the antigen sometimes occurred. As a negative control, triplicate sections were immunostained without exposure to primary antibody, which confirmed the staining specificity. To quantify the state of POFUT1 protein expression in those components, we used IHC scoring systems described previously $(6,8-17)$. The intensity of the POFUT1 immunoreaction was scored as follows: $1+$, weak; $2+$, moderate; and $3+$, intense. The cell numbers and the staining intensity then were multiplied to produce the POFUT1 IHC score. Cases with a POFUT1 IHC score exceeding 71.5 [the maximal score within +3 standard deviations (SD) of the mean of normal tissues] were defined as POFUT1-positive. The \pm 3 SD cutoff, which statistically is just $0.2 \%$ of the measurement and expected to fall outside this range, was used because it was unlikely to be affected by a random experimental error produced by sample manipulation (21). Two independent pathologists, neither of whom had knowledge of the patients' clinical status, made these judgments.

Transfection with shRNA plasmid. The OSCC cell lines, KOSC2 and HSC-2, in which POFUT1 protein expression was higher than in the other cell lines, were stably transfected with POFUT1 shRNA (shPOFUT1) or control shRNA (mock) (Santa Cruz Biotechnology) using Lipofectamine LTX and Plus Reagents (Invitrogen). The stable transfectants were isolated by the culture medium containing $1 \mathrm{mg} / \mathrm{ml}$ puromycin (Invitrogen). Two to 3 weeks after transfection, viable colonies were picked up and transferred to new dishes. shPOFUT1- and mock-transfected cells were used for further experiments. 
Proliferation assays. To investigate the effect of POFUT1 knockdown on cellular proliferation, shPOFUT1- and mocktransfected cells were seeded in 6-well plates at a density of $1 \times 10^{4}$ viable cells/well. At the indicated time-points, the cells were trypsinized and counted in triplicate using a hemocytometer and Cell Counting Kit-8 (Dojindo Molecular Technologies, Kumamoto, Japan) (22). Images were obtained every $12 \mathrm{~h}$ using a Leica LCD microscope (Leica Microsystems, Wetzlar, Germany) (original magnification, x200) and were representative of three independent experiments.

Migration assay. To evaluate the effect of POFUT1 knockdown on migration, we performed the wound-healing assay described previously (17). Briefly, after uniform wounds were made in confluent culture of the shPOFUT1 and mock cells, the extent of closure was monitored visually every $4 \mathrm{~h}$ for $24 \mathrm{~h}$. The results were visualized by measuring the wound spaces using Lenalaf220 software (available at http://www.vector.co.jp/ download/file/win95/art/fh442375.html). The mean value was calculated from data obtained from three separate chambers.

Invasiveness assay. To evaluate the effect of POFUT1 knockdown on invasiveness, we performed an invasiveness assay (23). A total of $2.5 \times 10^{5}$ cells were seeded on a polyethylene terephthalate membrane insert (pore size, $3 \mu \mathrm{m}$ ) in a transwell apparatus (Becton-Dickinson, Franklin Lakes, NJ, USA). In the lower chamber, $1 \mathrm{ml}$ of DMEM with 10\% FBS and puromycin (Invitrogen) were added as chemoattractants. After the cells were incubated for $22 \mathrm{~h}$ at $37^{\circ} \mathrm{C}$, the insert was washed with PBS and the cells on the top of the insert surface were removed with a cotton swab. Cells adhering to the lower surface of the membrane were fixed with methanol and stained with crystal violet; the numbers of cells invading the pores in five random fields were counted using a light microscope at x100 magnification.

Statistical analysis. The significance of the POFUT1 expression levels was evaluated using Fisher's exact test or the Mann-Whitney U test. $\mathrm{P}<0.05$ was considered statistically significant. The data are expressed as the mean \pm standard error of the mean (SEM).

\section{Results}

Evaluation of POFUT1 expression in OSCC-derived cell lines. We examined POFUT1 mRNA and protein expression in five OSCC-derived cell lines (Ho-1-u-1, KOSC2, HSC-4, HSC-3 and HSC-2) and HNOKs using qRT-PCR and western blot analysis. Significant upregulation of POFUT1 mRNA occurred in all OSCC cell lines compared with the HNOKs (Fig. 1A, $\mathrm{P}<0.05$ ).

Fig. 1B shows representative results of the western blot analyses. The values obtained from densitometric analysis of POFUT1 protein were normalized to the GAPDH levels and then expressed as a percentage of the HNOK values. A significant increase in POFUT1 protein expression was seen in all OSCC cell lines compared with the HNOKs (Fig. 1B).

Evaluation of POFUT1 expression in primary OSCCs. We evaluated POFUT1 mRNA expression in the normal
A

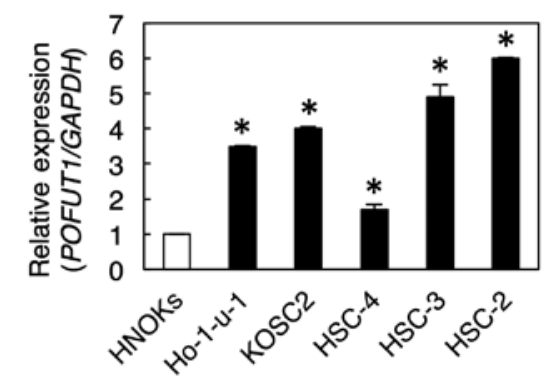

B

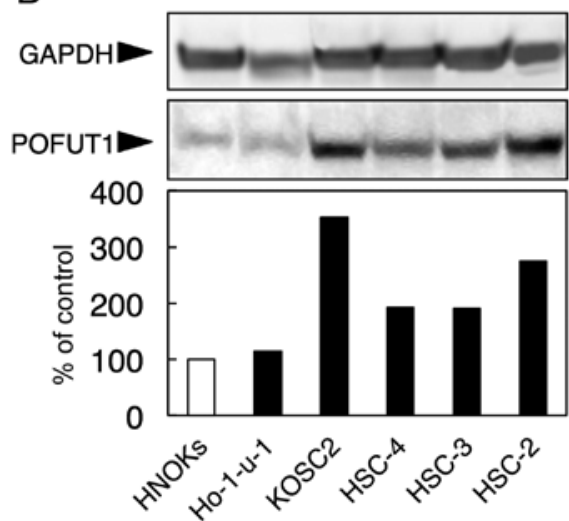

Figure 1. Evaluation of POFUT1 expression in OSCC-derived cell lines (A) POFUT1 mRNA levels are analyzed in OSCC-derived cells and HNOKs by qRT-PCR. Significant upregulation of POFUT1 mRNA is seen in five OSCC-derived cell lines compared with HNOKs $\left({ }^{*} \mathrm{P}<0.05\right.$, MannWhitney $U$ test). Data are expressed as the means \pm SEM in triplicate. (B) Representative western blot data of POFUT1 in OSCC-derived cell lines and HNOKs. Densitometric POFUT1 protein data are normalized to GAPDH protein levels. The values are expressed as a percentage of the HNOKs. POFUT1 protein is upregulated in OSCC-derived cell lines compared with HNOKs.

oral tissues and paired primary OSCCs from 128 patients. Similar to the data obtained from the OSCC-derived cell lines, qRT-PCR showed that POFUT1 mRNA expression was upregulated in 104 (81.2\%) of 128 primary OSCCs compared with the matched normal oral tissues (data not shown).

Representative IHC results for POFUT1 protein in normal oral tissue and primary OSCC are shown in Fig. 2A. The POFUT1 IHC scores of the cytoplasm in primary OSCCs were significantly $(\mathrm{P}<0.05)$ higher than in normal tissues. The IHC scores in the normal oral tissues and OSCCs ranged from 76.2 to 180.0 (median, 165.8) and 112.5 to 225.5 (median, 192.4), respectively (Fig. 2B). The POFUT1 IHC scores of T3/T4 were significantly $(\mathrm{P}<0.05)$ higher than that of $\mathrm{T} 1 / \mathrm{T} 2$. The IHC scores for T1/T2 and T3/T4 ranged from 77.8 to 215 (median, 189.5) and 149.8 to 240 (median, 204.5), respectively (Fig. 2C). Table I shows the correlations between the clinicopathologic characteristics of the patients with OSCC and the status of POFUT1 protein expression using the IHC scoring system. Among the clinical classifications, POFUT1-positive OSCCs were correlated with primary tumor size $(\mathrm{P}=0.048)$ (Table I).

Establishment of POFUT1 knockdown cells. Since POFUT1 expression was upregulated in the OSCC cell lines, we assumed 
Table I. Correlation between expression of POFUT1 and clinical parameters in OSCCs.

\begin{tabular}{|c|c|c|c|c|}
\hline \multirow[b]{3}{*}{ Clinical parameters } & \multicolumn{4}{|c|}{ No. of patients } \\
\hline & \multirow[b]{2}{*}{ Total } & \multicolumn{2}{|c|}{ Results of immunostaining } & \multirow[b]{2}{*}{ P-value } \\
\hline & & POFUT1-negative & POFUT1-positive & \\
\hline \multicolumn{5}{|l|}{ Age at surgery (years) } \\
\hline$<60$ & 52 & 14 & 38 & \multirow[t]{3}{*}{0.767} \\
\hline $60-70$ & 50 & 11 & 39 & \\
\hline$>70$ & 26 & 5 & 21 & \\
\hline \multicolumn{5}{|l|}{ Gender } \\
\hline Male & 70 & 15 & 55 & \multirow[t]{2}{*}{0.676} \\
\hline Female & 58 & 15 & 43 & \\
\hline \multicolumn{5}{|l|}{ T-primary tumor size } \\
\hline $\mathrm{T} 1$ & 27 & 7 & 20 & \multirow[t]{4}{*}{$0.048^{\mathrm{a}}$} \\
\hline $\mathrm{T} 2$ & 49 & 17 & 32 & \\
\hline $\mathrm{T} 3$ & 28 & 4 & 24 & \\
\hline $\mathrm{T} 4$ & 24 & 2 & 22 & \\
\hline $\mathrm{T} 1 / \mathrm{T} 2$ & 76 & 24 & 52 & \multirow[t]{2}{*}{$0.010^{\mathrm{a}}$} \\
\hline $\mathrm{T} 3 / \mathrm{T} 4$ & 52 & 6 & 46 & \\
\hline \multicolumn{5}{|c|}{ N-regional lymph node metastasis } \\
\hline $\mathrm{N}(+)$ & 68 & 15 & 53 & \multirow[t]{2}{*}{0.835} \\
\hline $\mathrm{N}(-)$ & 60 & 15 & 45 & \\
\hline \multicolumn{5}{|l|}{ Stage } \\
\hline I & 29 & 5 & 24 & \multirow[t]{4}{*}{0.128} \\
\hline II & 42 & 14 & 28 & \\
\hline III & 29 & 8 & 21 & \\
\hline IV & 28 & 3 & 25 & \\
\hline $\mathrm{I} / \mathrm{II}$ & 71 & 19 & 52 & \multirow[t]{2}{*}{0.403} \\
\hline III/IV & 57 & 11 & 46 & \\
\hline \multicolumn{5}{|l|}{ Histopathological type } \\
\hline Well differentiated & 68 & 19 & 49 & \multirow[t]{3}{*}{0.400} \\
\hline Moderately differentiated & 55 & 10 & 45 & \\
\hline Poorly differentiated & 5 & 1 & 4 & \\
\hline \multicolumn{5}{|l|}{ Tumor site } \\
\hline Tongue & 46 & 10 & 36 & \multirow[t]{5}{*}{0.831} \\
\hline Gingiva & 38 & 11 & 27 & \\
\hline Buccal mucosa & 23 & 6 & 17 & \\
\hline Palate & 14 & 2 & 12 & \\
\hline Oral floor & 7 & 1 & 6 & \\
\hline Total & 128 & 30 & 98 & \\
\hline
\end{tabular}

${ }^{\mathrm{a}} \mathrm{P}<0.05$.

that POFUT1 might play an important role in OSCCs. To assess the POFUT1 functions in vitro, an shRNA experiment was performed using the KOSC 2 and HSC-2 cells. Expressions of POFUT1 mRNA and protein in the shPOFUT1-transfected cells were significantly $(\mathrm{P}<0.05)$ lower than those in the mocktransfected cells (KOSC2 and HSC-2 derived transfectant cells) (Fig. 3).
Functional analysis of POFUT1 knockdown cells. To evaluate the effect of POFUT1 knockdown on cellular growth, we performed a cellular proliferation assay. shPOFUT1- and mock-transfected cells were seeded in 6-well plates at a density of $1 \times 10^{4}$ viable cells/well counted on 7 consecutive days. There was a significant $(\mathrm{P}<0.05)$ decrease in cellular growth of the shPOFUT1-transfected cells compared with the 
A
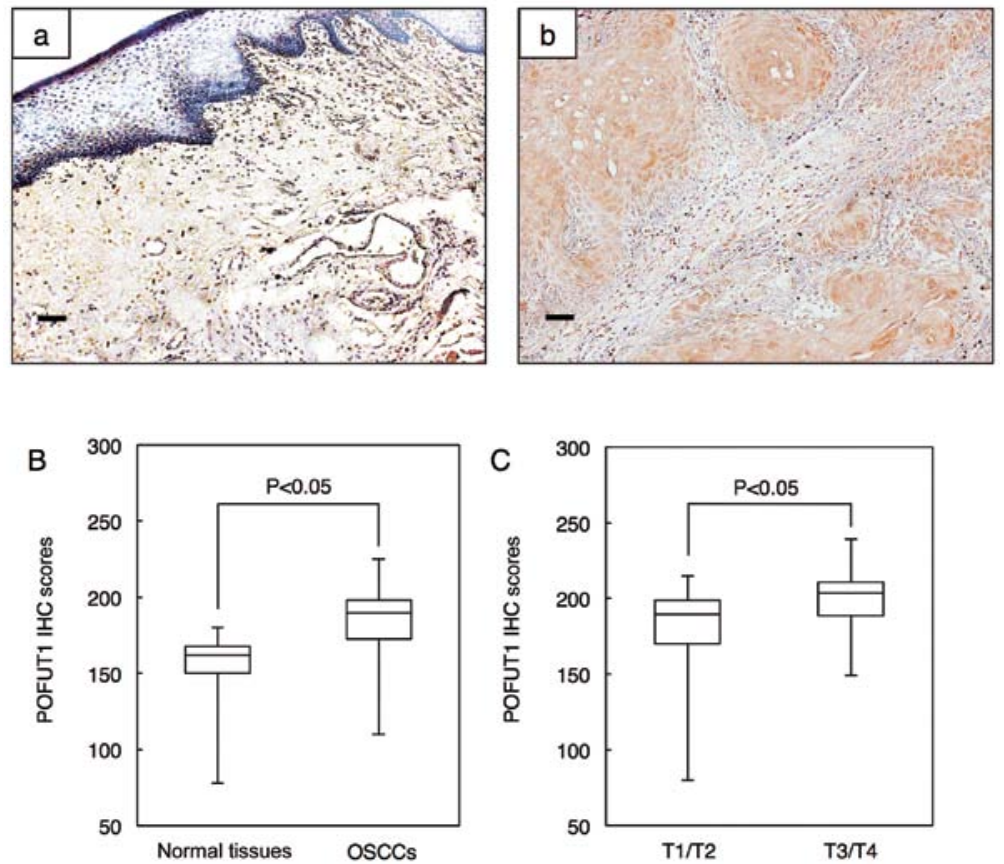

Figure 2. Evaluation of POFUT1 protein expression in primary OSCCs. (A) Representative IHC results of POFUT1 in normal oral tissue and primary OSCC. (a) Normal oral tissues exhibit negative POFUT1 protein expression. Skeletal muscle tissues are immunostained for POFUT1 (positive control). Original magnification, $\mathrm{x} 100$. Scale bars, $50 \mu \mathrm{m}$. (b) Positive immunoreactivity for POFUT1 in OSCCs is detected in the cytoplasm. Original magnification, x100. Scale bars, $50 \mu \mathrm{m}$. (B) State of POFUT1 protein expression in normal oral tissues $(\mathrm{n}=128)$ and primary OSCCs $(\mathrm{n}=128)$. The POFUT1 IHC scores are calculated as follows: IHC score $=1 \mathrm{x}$ (number of weakly stained cells in the field) $+2 \mathrm{x}$ (number of moderately stained cells in the field) $+3 \mathrm{x}$ (number of intensely stained cells in the field). The POFUT1 IHC scores for normal oral tissues and OSCCs range from 76.2 to 180.0 (median, 165.8) and 112.5 to 225.5 (median, 192.4), respectively. The POFUT1 protein expression level in OSCCs is significantly $(\mathrm{P}<0.05$, Mann-Whitney U test) higher than that in normal oral tissues. (C) The POFUT1 IHC scores for T3/T4 (149.8 to 240; median, 204.5) are significantly (P<0.05, Mann-Whitney U test) higher than those of T1/T2 (77.8 to 215; median, 189.5).

A
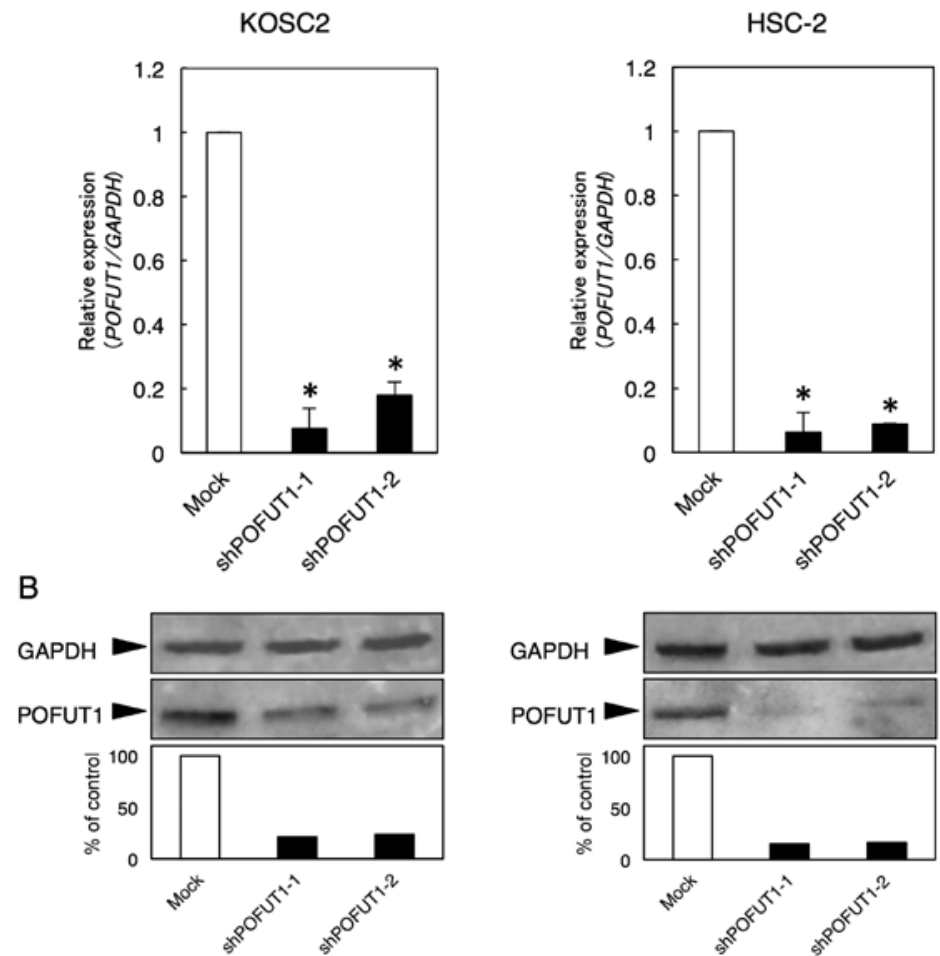

B

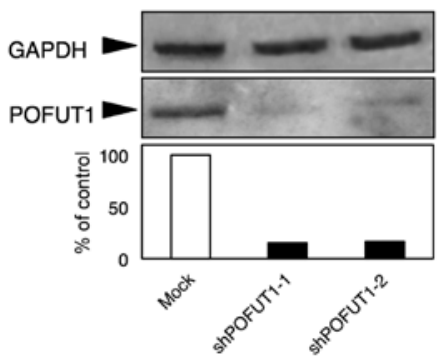

Figure 3. Expression of POFUT1 in shPOFUT1-transfected cells. (A) qRT-PCR shows that POFUT1 mRNA expression in the shPOFUT1-transfected cells (KOSC2- and HSC-2-derived tranfectant cells; two clones each) is significantly ( $\mathrm{P}<0.05$, Mann-Whitney $\mathrm{U}$ test) lower than that in the mock-transfected cells. (B) Western blot analysis shows that the POFUT1 protein levels in the shPOFUT1-transfected cells (KOSC2- and HSC-2-derived transfectant cells; two clones each) also have decreased markedly compared with that in the mock-transfected cells. 
A

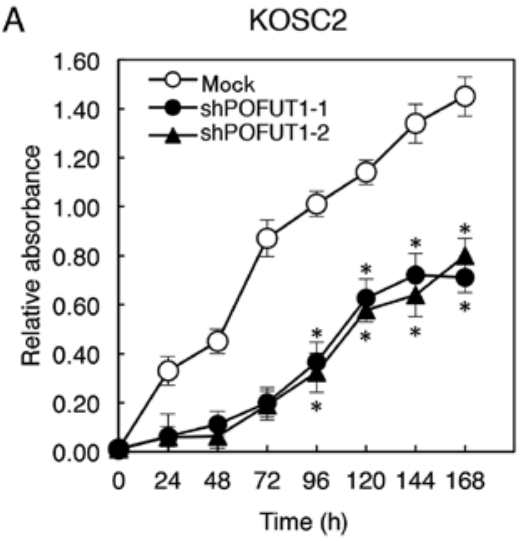

B

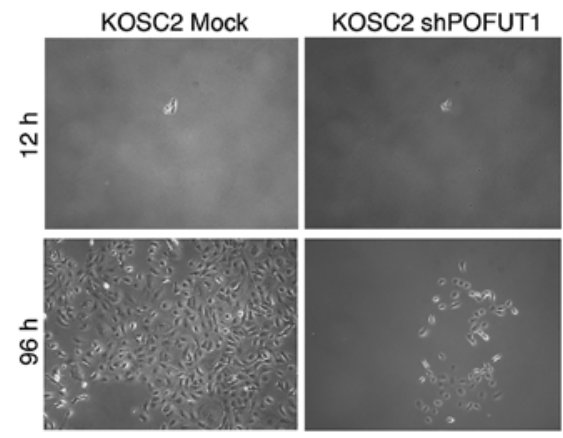

HSC-2

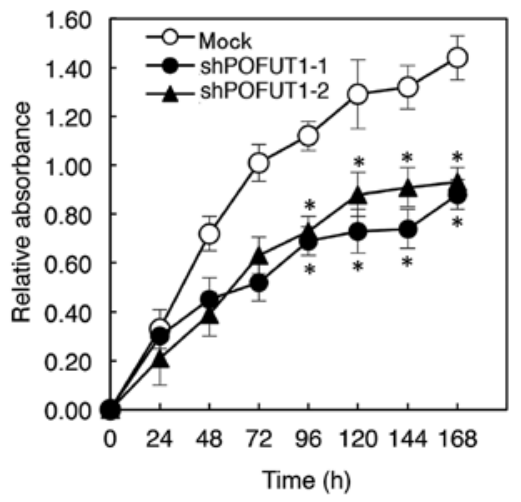

HSC-2 Mock

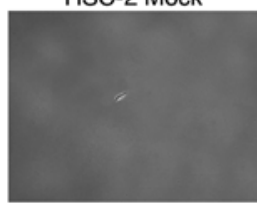

HSC-2 shPOFUT1
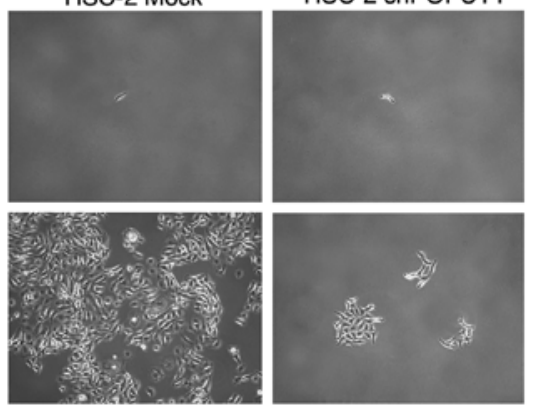

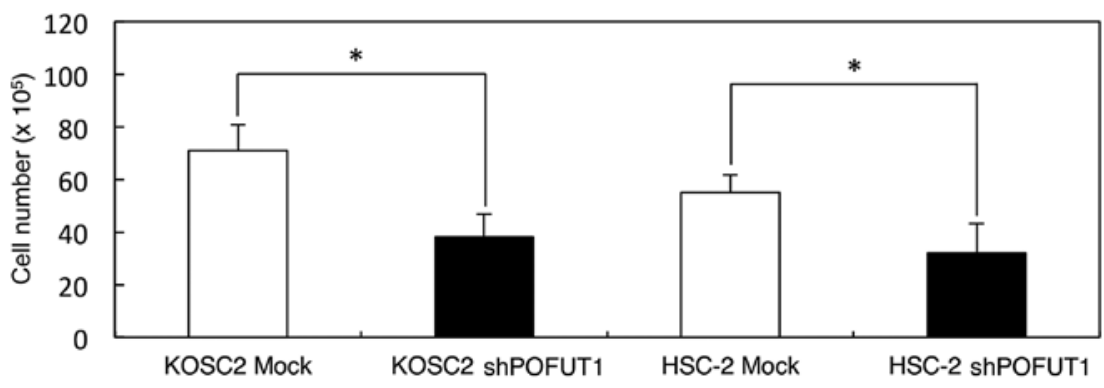

Figure 4. Proliferation of shPOFUT1-transfected cells. (A) To determine the effect of shPOFUT1 on cellular proliferation, shPOFUT1- and mock-transfected cells (KOSC2- and HSC-2-derived transfectant cells) are seeded in 6-well plates at a density of 1x10 ${ }^{4}$ viable cells/well. shPOFUT1- and mock-transfected cells were counted on 7 consecutive days using Cell Counting Kit-8. The growth of shPOFUT1-transfected cells is inhibited significantly compared with that of the mock-transfected cells after 7 days. The results are expressed as the means \pm SEM of values from three assays. The asterisks indicate significant differences between the shPOFUT1- and mock-transfected cells ("P $<0.05$, Mann-Whitney U test). (B) shPOFUT1- and mock-transfected cells were counted on 4 consecutive days by a hemocytometer. Images were obtained after 12 and $96 \mathrm{~h}$ using a Leica LCD microscope (original magnification, x200) and are representative of three independent experiments. The number of shPOFUT1-transfected cells is significantly ( $\mathrm{P}<0.05)$ decreased compared with that of mock-transfected cells after $96 \mathrm{~h}$ (KOSC2- and HSC-2-derived transfectant cells).

mock-transfected cells (KOSC2 and HSC-2 derived transfectant cells) (Fig. 4A). Photomicrographs (Fig. 4B) showed the cytostatic effect $(\mathrm{P}<0.05)$ of cell line-transfected shPOFUT1 and mock cells. In the migration assay, when we visually monitored the uniform wound area in confluent cell culture, the wound areas in POFUT1 knockdown cells were the same as that in the mock cells. In the invasiveness assay, the number of penetrating POFUT1 knockdown cells was similar to that of the mock cells (data not shown).

\section{Discussion}

We previously reported the gene expression profiling of OSCC to identify genes associated with oral carcinogenesis (6). Using microarray analysis, POFUT1 was one of the most markedly upregulated genes in OSCC-derived cell lines.
POFUT1, located on chromosome 20q11.21, produces an $O$-fucose modification on EGF-like repeats of a number of cellular surface and secreted proteins (7). Recent studies have reported that POFUT1 affected anterior-posterior somite patterning in mammalian embryos (24) and regulated T-, myeloid- and B-lineage differentiation in mammals (25). Several laboratories have reported the relationship between POFUT1 expression and malignancies, such as acute myeloid leukemia, myeloid dysplastic syndrome, glioblastoma and colon cancer (26-29). Taken together, we anticipated that POFUT1 may play an important role in various tumoral behavior.

The present study showed significant upregulation of POFUT1 levels in OSCCs and five OSCC-derived cell lines compared with the matched normal counterparts. We investigated the relationship between POFUT1 expression 
and clinical behavior of patients with OSCC. The POFUT1 protein expression levels in primary OSCCs were associated significantly with tumoral size. The POFUT1 IHC scores of T3/T4 were much higher than those of T1/T2. These results suggested a strong association between POFUT1 and progression of OSCC. We also examined the effect of POFUT1 on cellular proliferation. POFUT1 knockdown in OSCC-derived cells resulted in obvious reduction of growth. A migration assay showed that the uniform wound area in POFUT1 knockdown cell was the same as that in the mock cells. An invasiveness assay indicated that the number of penetrating POFUT1 knockdown cells was similar to that of the mock cells. These results are consistent with the association between POFUT1 level and lymph node metastasis in OSCC (Table I).

In conclusion, POFUT1 is overexpressed frequently in OSCCs and could play an important role in the course of cellular proliferation. POFUT1 is likely to be not only a molecular marker for tumoral growth but also an efficacious treatment strategy for preventing progression in OSCCs.

\section{Acknowledgements}

We thank Ms. Lynda C. Charters for editing this manuscript.

\section{References}

1. Severino P, Alvares AM, Michaluart P Jr, Okamoto OK, Nunes FD, Moreira-Filho CA and Tajara EH: Global gene expression profiling of oral cavity cancers suggests molecular heterogeneity within anatomic subsites. BMC Res Notes 1: 113-121, 2008.

2. Scully C, Field JK and Tanzawa H: Genetic aberrations in oral or head and neck squamous cell carcinoma (SCCHN): 1. Carcinogen metabolism, DNA repair and cell cycle control. Oral Oncol 36: 256-263, 2000.

3. Scully C, Field JK and Tanzawa H: Genetic aberrations in oral or head and neck squamous cell carcinoma 3: clinico-pathological applications. Oral Oncol 36: 404-413, 2000.

4. Sticht C, Freier K, Knöpfle K, Flechtenmacher C, Pungs S, Hofele C, Hahn M, Joos S and Lichter P: Activation of MAP kinase signaling through ERK5 but not ERK1 expression is associated with lymph node metastases in oral squamous cell carcinoma (OSCC). Neoplasia 10: 462-470, 2008.

5. Macfarlane GJ, Zheng T, Marshall JR, Boffetta P, Niu S, Brasure J, Merletti F and Boyle P: Alcohol, tobacco, diet and the risk of oral cancer: a pooled analysis of three case-control studies. Eur J Cancer B Oral Oncol 31B: 181-187, 1995.

6. Yamano Y, Uzawa K, Shinozuka K, Fushimi K, Ishigami T, Nomura H, Ogawara K, Shiiba M, Yokoe $\mathrm{H}$ and Tanzawa $\mathrm{H}$ : Hyaluronan-mediated motility: a target in oral squamous cell carcinoma. Int J Oncol 32: 1001-1009, 2008.

7. Wang Y, Lee GF, Kelley RF and Spellman MW: Identification of a GDP-L-fucose: polypeptide fucosyltransferase and enzymatic addition of $O$-linked fucose to EGF domains. Glycobiology 6 : 837-842, 1996.

8. Koike H, Uzawa K, Grzesik WJ, Seki N, Endo Y, Kasamatsu A, Yamauchi M and Tanzawa H: GLUT1 is highly expressed in cementoblasts but not in osteoblasts. Connect Tissue Res 46: $117-124,2005$.

9. Kasamatsu A, Uzawa K, Nakashima D, Koike H, Shiiba M, Bukawa H, Yokoe H and Tanzawa H: Galectin-9 as a regulator of cellular adhesion in human oral squamous cell carcinoma cell lines. Int J Mol Med 16: 269-273, 2005.

10. Endo Y, Uzawa K, Mochida Y, Shiiba M, Bukawa H, Yokoe H and Tanzawa H: Sarcoendoplasmic reticulum $\mathrm{Ca}(2+)$ ATPase type 2 downregulated in human oral squamous cell carcinoma. Int J Cancer 110: 225-231, 2004.

11. Sakuma K, Kasamatsu A, Yamatoji M, Yamano Y, Fushimi K, Iyoda M, Ogoshi K, Shinozuka K, Ogawara K, Shiiba M, Tanzawa $\mathrm{H}$ and Uzawa $\mathrm{K}$ : Expression status of Zic family member 2 as a prognostic marker for oral squamous cell carcinoma. J Cancer Res Clin Oncol 136: 553-559, 2010.
12. Yamatoji M, Kasamatsu A, Kouzu Y, Koike H, Sakamoto Y, Ogawara K, Shiiba M, Tanzawa H and Uzawa K: Dermatopontin: a potential predictor for metastasis of human oral cancer. Int J Cancer 130: 2903-2911, 2012

13. Yamatoji M, Kasamatsu A, Yamano Y, Sakuma K, Ogoshi K, Iyoda M, Shinozuka K, Ogawara K, Takiguchi Y, Shiiba M, Tanzawa H and Uzawa K: State of homeobox A10 expression as a putative prognostic marker for oral squamous cell carcinoma. Oncol Rep 23: 61-67, 2010.

14. Kato Y, Uzawa K, Yamamoto N, Kouzu Y, Koike H, Shiiba M, Bukawa H, Yokoe H, Shibahara T and Tanzawa H: Overexpression of Septin1: possible contribution to the development of oral cancer. Int J Oncol 31: 1021-1028, 2007.

15. Kouzu Y, Uzawa K, Koike H, Saito K, Nakashima D, Higo M, Endo Y, Kasamatsu A, Shiiba M, Bukawa H, Yokoe H and Tanzawa H: Overexpression of stathmin in oral squamous-cell carcinoma: correlation with tumour progression and poor prognosis. Br J Cancer 94: 717-723, 2006.

16. Iyoda M, Kasamatsu A, Ishigami $T$, Nakashima D, EndoSakamoto Y, Ogawara K, Shiiba M, Tanzawa H and Uzawa K: Epithelial cell transforming sequence 2 in human oral cancer. PLoS One 5: e14082, 2010.

17. Onda T, Uzawa K, Nakashima D, Saito K, Iwadate Y, Seki N, Shibahara T and Tanzawa H: Lin-7C/VELI3/MALS-3: an essential component in metastasis of human squamous cell carcinoma. Cancer Res 67: 9643-9648, 2007.

18. Saito Y, Kasamatsu A, Yamamoto A, Shimizu T, Yokoe H, Sakamoto Y, Ogawara K, Shiiba M, Tanzawa H and Uzawa K: ALY as a potential contributor to metastasis in human oral squamous cell carcinoma. J Cancer Res Clin Oncol 139: 585-594, 2013.

19. Koike K, Kasamatsu A, Iyoda M, Saito Y, Kouzu Y, Koike H, Sakamoto Y, Ogawara K, Tanzawa $\mathrm{H}$ and Uzawa K: High prevalence of epigenetic inactivation of the human four and a half LIM domains 1 gene in human oral cancer. Int J Oncol 42: 141-150, 2013.

20. Usukura K, Kasamatsu A, Okamoto A, Kouzu Y, Higo M, Koike H, Sakamoto Y, Ogawara K, Shiiba M, Tanzawa H and Uzawa K: Tripeptidyl peptidase II in human oral squamous cell carcinoma. J Cancer Res Clin Oncol 139: 123-130, 2013.

21. Verburg FA, Wäschle K, Reiners C, Giovanella L and Lentjes EG: Heterophile antibodies rarely influence the measurement of thyroglobulin and thyroglobulin antibodies in differentiated thyroid cancer patients. Horm Metab Res 42: 736-739, 2010.

22. Imam JS, Buddavarapu K, Lee-Chang JS, Ganapathy S, Camosy C, Chen Y and Rao MK: MicroRNA-185 suppresses tumor growth and progression by targeting the Six 1 oncogene in human cancers. Oncogene 29: 4971-4979, 2010.

23. Ogoshi K, Kasamatsu A, Iyoda M, Sakuma K, Yamatoji M, Sakamoto Y, Ogawara K, Shiiba M, Tanzawa H and Uzawa K: Dickkopf-1 in human oral cancer. Int J Oncol 39: 329-336, 2011.

24. Schuster-Gossler K, Harris B, Johnson KR, Serth J and Gossler A: Notch signalling in the paraxial mesoderm is most sensitive to reduced POFUT1 levels during early mouse development. BMC Dev Biol 9: 6, 2009.

25. Stanley $P$ and Guidos CJ: Regulation of Notch signaling during T- and B-cell development by $O$-fucose glycans. Immunol Rev 230: 201-215, 2009.

26. Gurvich N, Perna F, Farina A, Voza F, Menendez S, Hurwitz J and Nimer SD: L3MBTL1 polycomb protein, a candidate tumor suppressor in del(20q12) myeloid disorders, is essential for genome stability. Proc Natl Acad Sci USA 107: 22552-22557, 2010.

27. Mackinnon RN, Selan C, Wall M, Baker E, Nandurkar H and Campbell LJ: The paradox of 20q11.21 amplification in a subset of cases of myeloid malignancy with chromosome 20 deletion. Genes Chromosomes Cancer 49: 998-1013, 2010.

28. Kroes RA, Dawson G and Moskal JR: Focused microarray analysis of glyco-gene expression in human glioblastomas. J Neurochem 103 (Suppl 1): 14-24, 2007.

29. Loo LW, Tiirikainen M, Cheng I, Lum-Jones A, Seifried A, Church JM, Gryfe R, Weisenberger DJ, Lindor NM, Gallinger S, Haile RW, Duggan DJ, Thibodeau SN, Casey G and Le Marchand L: Integrated analysis of genome-wide copy number alterations and gene expression in microsatellite stable, $\mathrm{CpG}$ island methylator phenotype-negative colon cancer. Genes Chromosomes Cancer 52: 450-466, 2013. 